\title{
In vivo anti-staphylococcal activity of roselle (Hibiscus sabdariffa L.) calyx extract in Drosophila model of infection
}

\author{
Muhammad Ahsan ${ }^{1,2}$, Alvin Valentino Gonsales ${ }^{1}$, Sartini Sartini ${ }^{1}$, Elly Wahyudin $^{1}$, Firzan Nainu $^{1 *}$ \\ ${ }^{1}$ Faculty of Pharmacy, Hasanuddin University, Makassar, South Sulawesi, Indonesia \\ ${ }^{2}$ Akademi Farmasi Yamasi, Makassar, South Sulawesi, Indonesia
}

\section{A R T I C L E I N F O}

Article Type:

Original Article

Article History:

Received: 19 September 2018

Accepted: 9 November 2018

\section{Keywords:}

Antibacterial agent

Staphylococcus aureus

Roselle

Fruit flies

\begin{abstract}
A B S T R A C T
Introduction: The emergence of antibiotic-resistant Staphylococcus aureus is a major threat for worldwide communities. To overcome such serious problem, the discovery of novel antibacterial agents through exploration of diverse potential sources is essential. The aim of this research was to investigate the in vivo anti-staphylococcal activity of roselle (Hibiscus sabdariffa L.) calyx extract against $S$. aureus in fruit flies (Drosophila melanogaster) model of infection.

Methods: Roselle calyces were dried and extracted with ethanol using maceration method. Wildtype fruit flies were infected with $S$. aureus and subjected to survival assay, bacterial load examination, and gene expression analysis, in the presence or absence of roselle calyx extract. Survival and bacterial load analysis were subsequently performed on immunodeficient fruit flies using similar protocols.

Results: Reduction of host survivorship accompanied by increasing level of bacterial proliferation was observed in group of wildtype fruit flies infected with $S$. aureus. These phenotypic events were further augmented in mutant flies devoid of component for antibacterial immune responses. Nevertheless, reduction of bacterial load and improvement of host survival were demonstrated in either wildtype or immunodeficient fruit flies upon treatment with roselle calyx extract after bacterial challenge, irrespective of immune status.

Conclusion: Collectively, our results demonstrated the in vivo antibacterial activity of roselle calyx extract against $S$. aureus in Drosophila model of infection and this was not due to induction of immune response in the host.
\end{abstract}

Implication for health policy/practice/research/medical education:

Our results demonstrated the in vivo antibacterial activity of roselle calyx extract against Staphylococcus aureus using a novel Drosophila infection model platform that shall emphasize the idea to explore the use of roselle calyx extract as a source to discover novel anti-staphylococcal agents.

Please cite this paper as: Ahsan M, Gonzalez AV, Sartini S, Wahyudin E, Nainu F. In vivo anti-staphylococcal activity of roselle (Hibiscus sabdariffa L.) calyx extract in Drosophila model of infection. J Herbmed Pharmacol. 2018;9(1):41-46. doi: 10.15171/ jhp.2019.07.

\section{Introduction}

Antibiotics are considered as the most used class of drugs in the world. Between 2000 and 2010, the consumption of antibiotics reached more than $30 \%$ of the total use of medicines worldwide (1). In Indonesia itself, the utilization of antibiotics even reached $80 \%$ of total drugs consumed between 2002 to 2005 (2). Unfortunately, in some cases, antibiotics were purchased in the absence of proper examination and decision from physician or even without the needs of prescription, implicating the irrational practice of antibiotics utilization. Consequently, in addition to other contributing factors, the emergence of antibiotic-resistant bacteria has been constantly reported $(1,3,4)$.

The emergence of bacterial strains that are resistant to antibiotics is a major concern for public health (3-5). One of the pathogenic bacteria that presents serious threat to human population is Staphylococcus aureus (6), especially after the emergence of methicillin-resistant $S$. aureus (MRSA) (7). To overcome this life-threatening problem, the discovery of novel antibacterial agents through exploration of diverse potential sources is essential (4, 
8). One of promising sources for such efforts is roselle (Hibiscus sabdariffa L.). The ethanolic extract of roselle's calyx has been shown to yield excellent in vitro antibacterial effect against several Gram-positive pathogenic bacteria (9-11), including S. aureus and MRSA $(12,13)$. However, despite such promising antibacterial effect, no in vivo experiment has been carried out to further clarify the antistaphylococcal activity of roselle calyx ethanolic extract.

Recently, we introduced an inexpensive in vivo platform to assess the anti-staphylococcal activity of green algae Ulva reticulata (14). In this platform, we used wild type and immunodeficient fruit fly (Drosophila melanogaster) as host organisms in S. aureus infection and made use of two simple phenotypic assays: survival assay and determination of bacterial load (14). To date, D. melanogaster has been recognized as a pioneer model organism in the revelation of important signaling pathways related to immunity against not only bacterial infection $(15,16)$ but also viral infection (17-19). In addition to that, D. melanogaster has been introduced as one of emerging human disease models $(20,21)$ and considered as one of suitable model organisms in the drug discovery (22-25).

Drosophila melanogaster offers great advantages as an in vivo model system in antibacterial drug discovery research. In addition to the fact that it has been shown as an appropriate host for $S$. aureus (14,26-28), D. melanogaster shares high degree of genetic similarity with human (up to $75 \%$ ) (21) which suggests high possibility for getting the same results upon clinical trial on human subjects. Moreover, the use of fruit flies as a model organism raises almost no ethical issues and requires little effort in the stock maintenance $(20,21,29)$. Based on such advantages, we carried out this research to investigate the in vivo antistaphylococcal activity of roselle extract against $S$. aureus in fruit flies model of infection.

\section{Materials and Methods}

Bacterial strains and fly stocks

Fresh culture of S. aureus ATCC 29213 strain was used as an infectious agent in the entire experiments. To obtain the appropriate inoculum, the bacteria was cultured in Nutrient Broth $(\mathrm{NB})$ medium at $37^{\circ} \mathrm{C}$ for $1 \times 24$ hours, collected and washed thoroughly with PBS followed by quantification using spectrophotometry method. The inoculum of $S$. aureus was suspended in PBS prior to use in the experiments. The following lines of Drosophila were used in this study: $w^{1118}$ as genotype (background) control and $s p z^{r m 7 / T M 6}$ as immunodeficient fly line (gifts from Prof. Yoshinobu Nakanishi, Kanazawa University) which has impaired activity of Spätzle. All fly lines were maintained with standard cornmeal-agar medium at $25^{\circ} \mathrm{C}$.

\section{Extract preparation}

Samples of roselle (Hibiscus sabdariffa L.) calyces were obtained from Makassar area, South Sulawesi, Indonesia.
These samples were sorted and subjected to maceration procedure using 96\% ethanol for 1 x 24 hours and remacerated for 1 x 24 hours. All filtrates were collected and further processed using rotavapor until extract with appropriate thickness were obtained. The resulting extract was stored in a brown silica container.

Fly infection and assays for survival and bacterial growth Infection experiment was carried out on either background control or immunodeficient fly line using pricking method, in which bacteria was introduced into the thorax of male adult flies, as described previously (14). In brief, flies at age of 4-7 days after eclosion (10 flies per vial, 3 vials in each experiment) were subjected to bacterial pricking $\left(6 \times 10^{5} \mathrm{cfu} / \mathrm{mL}\right.$ per fly) and further maintained at $29^{\circ} \mathrm{C}$. Pricked flies were then subjected to either survival assay or colony forming assay as described previously (14). For the survival assay, the flies were pricked with $S$. aureus and maintained in vials in the presence or absence of treatments and number of live flies at each group was recorded daily during experiment. For the colony forming assay, flies were pricked with $S$. aureus and maintained in vials in the presence or absence of treatments. At a given time, live flies were taken from each group and mechanically processed using a micropestle in PBS solution. Homogenates obtained from each group were subsequently prepared at a serial dilution and then plated on Vogel-Johnson agar medium. Number of colonies appeared after incubation was expressed as colony-forming unit (cfu) per ml. Groups of healthy flies pricked with PBS were used as controls in both survival and colony forming assays.

\section{Gene expression analysis}

Total RNA was extracted from five live Drosophila harvested from each group of treatments at 48 hours post infection and homogenized manually in the Treff tube using Micropestle followed by further processing using SV Total RNA Isolation System (Promega) according to the manufacturer's protocol. Analysis of drosomycin (drs) level was carried out using one set of $d r s$ primer (sequence of $d r s$ forward primer: $5^{\prime}$ - CGT GAG AAC CTT TTC CAA TAT GAT G - 3' and sequence of drs reverse primer: $5^{\prime}$ TCC CAG GAC CAC CAG CAT - 3') in a $20 \mu$ reaction volume using GoTaq 1 -Step RT-qPCR System (Promega), as per manufacturer's instruction. Ribosomal protein rp49 was used as host reference gene and examined by using one set of $r p 49$ primer (sequence of $r p 49$ forward primer: 5' - GAC GCT TCA AGG GAC AGT ATC TG - 3' and sequence of rp49 reverse primer: 5' - AAA CGC GGT TCT GCA TGA G - 3') using the same protocol. RotorGene Q thermal cycler (Qiagen, Germany) was used with the following profile: $37^{\circ} \mathrm{C}$ for 15 minutes, $95^{\circ} \mathrm{C}$ for 10 minutes, and 40 cyclic repeats of $95^{\circ} \mathrm{C}$ for 10 seconds and $60^{\circ} \mathrm{C}$ for 30 seconds, and $72^{\circ} \mathrm{C}$ for 30 seconds. A 
standard melt curve analysis was carried out to confirm that only the expected product had been amplified. The abundance of $d r s$ relative to the host reference gene $r p 49$ was determined using qGENE software.

Data processing and statistical analysis

Results obtained from at least three independent biological replicates were processed using GraphPad Prism ${ }^{\circledR} 7$. Survival curve was prepared as a Kaplan-Meier curve and followed by log-rank analysis. For analysis of CFU and mRNA level of $d r s$, data were prepared as bar graphs and analyzed statistically using one-way ANOVA. For all statistical analysis, data were presented as mean \pm S.D and $P$ values of less than 0.05 were considered significant.

\section{Results}

Dose-dependent toxicity of roselle calyx extract in adult Drosophila melanogaster

In this experiment, we used in vivo Drosophila platform to assess the antibacterial effect of roselle on S. aureus. Prior to antibacterial activity assessment, we carried out a simple survival assay to determine the safest concentration of roselle calyx extract to be used in the further experiments. As shown in Figure 1, D. melanogaster $w^{1118}$ (Figure 1A) and $s p z$ mutant line (Figure $1 \mathrm{~B}$ ) were succumbed to immediate and gradual death upon ingestion of roselle calyx extract at high concentrations $(20 \%$ and $10 \% \mathrm{w} / \mathrm{w}$, respectively). However, the survival of flies was seemingly
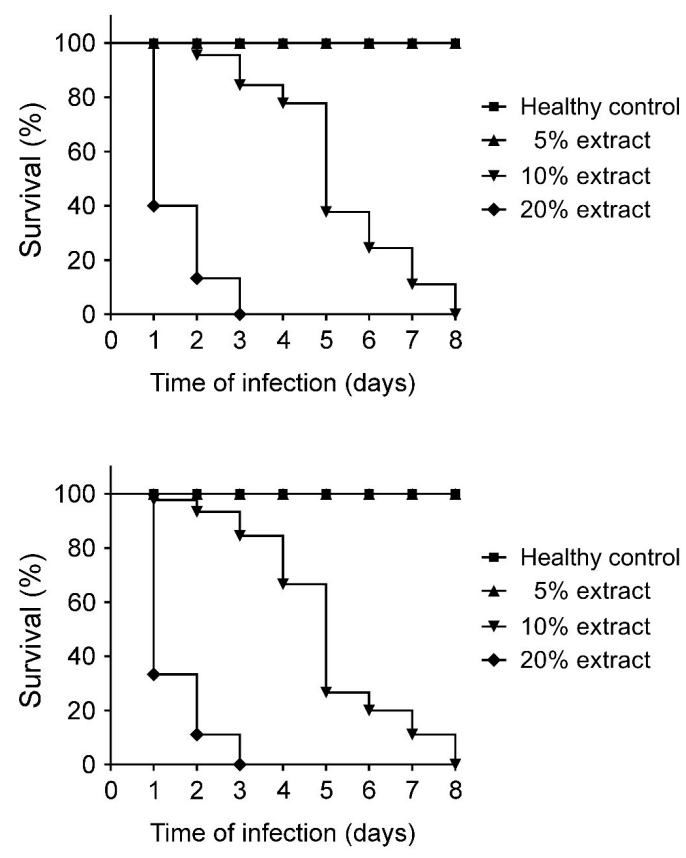

Figure 1. Ingestion of roselle calyx extract reduced the survival of $D$. melanogaster in a concentration-dependent manner. Adult flies $w^{1118}(\mathrm{~A})$ and $s p z^{\text {rm7/TM6 }}(\mathrm{B})$ with age of 4-7 days were fed roselle calyx extract-containing food at three different concentrations $(5,10$, and $20 \%$ ) and incubated at $25^{\circ} \mathrm{C}$. Number of dead flies were observed daily and fly survival was carried out using a Kaplan-Meier-Log Rank analysis. not affected once maintained in foods containing roselle calyx extracts at lower concentration $(5 \% \mathrm{w} / \mathrm{w})$, indicating the toxicity effect of this extract on $D$. melanogaster was occurred in a dose-dependent manner. Therefore, to rule out the possible influence of lethal effect of extract, we used roselle calyx extract at a concentration of $5 \%(\mathrm{w} / \mathrm{w})$ in further experiments.

Improvement of Drosophila melanogaster survival rate by roselle calyx extract under infection condition

Staphylococcus aureus is a gram-positive bacterium that has been shown to exert wide-ranging negative effect on many organisms including humans (31) and D. melanogaster (27). As shown in Figure 2A, infection of D. melanogaster $w^{1118}$ by $S$. aureus resulted in the reduction of flies' survival rate. However, treatment of S. aureus-infected Drosophila $w^{1118}$ with tetracycline, a potent bacteria protein synthesis inhibitor, was sufficient to prevent host early death phenotype, as documented by previous investigator (27) and recently confirmed by our group (14). In addition to that, treatment using ethanolic extract of roselle calyx at concentration of $5 \%$ was able to improve the survival of $S$. aureus-infected D. melanogaster, demonstrating the in vivo antibacterial activity of roselle calyx extract against $S$. aureus at the corresponding concentration.

Inhibition of Staphylococcus aureus growth by roselle calyx extract

Decreased survivorship of D. melanogaster upon S. aureus infection has been shown to be associated with increased
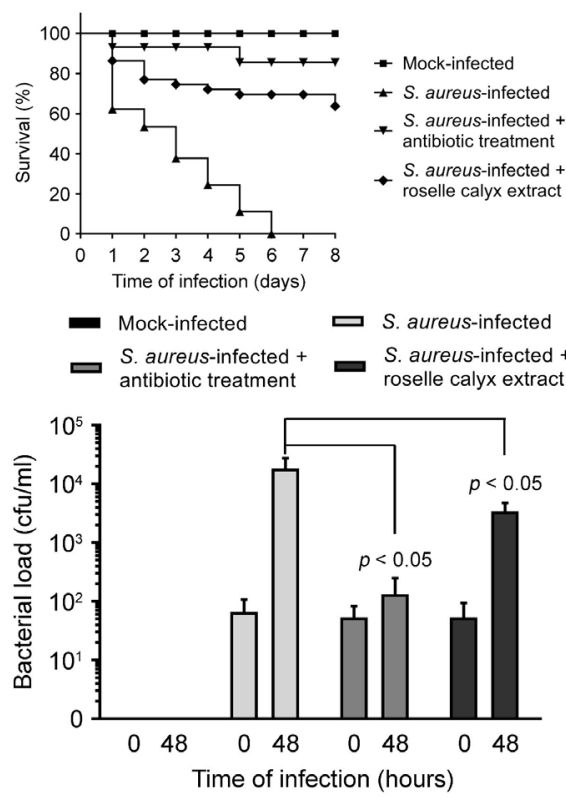

Figure 2. Improvement of host survival and reduced bacterial load in infected $w^{1118}$ flies in the presence roselle calyx extract. Adult $w^{1118}$ flies at 4-7 days after eclosion were infected with $6 \times 10^{5} \mathrm{cfu} / \mathrm{ml}$ of $S$. aureus by pricking, incubated at $25^{\circ} \mathrm{C}$ in the presence of $5 \%$ roselle calyx extract, and subjected to fly survival (A) and bacterial load (B) analysis. Flies treated with tetracycline at $200 \mu \mathrm{g} / \mathrm{ml}$ were used as a positive control group. 
bacterial load (27) and inhibition of bacterial growth by antibiotics improved the survival of infected host $(27,32)$. Taken this into account, we anticipated the reduction of bacterial load will be seen in the infected flies treated with roselle calyx extract. To examine this, we carried out a colony forming assay. As a result, we found that treatment of $S$. aureus-infected flies with either tetracycline or roselle calyx extract was certainly beneficial to reduce the bacterial load (Figure 2B), further supporting the notion that inhibition of bacterial growth by antibiotic or certain extract with potent antibacterial activity could lead to increased survivorship of infected flies.

Anti-staphylococcal activity of roselle calyx extract was not due to increased expression of antimicrobial peptide Drosomycin

Upon infection with gram-positive bacteria, Drosophila produced several antimicrobial peptides (AMP) that serve as part of humoral immune responses $(15,33)$. One of the well-characterized AMPs in Drosophila against grampositive bacteria such as $S$. aureus is called drosomycin (33), which is expressed by drs gene. To confirm whether drosomycin plays a role in the anti-staphylococcal activity of roselle extract, we examined the expression level of $d r s$ gene using RT-qPCR method. We found that the expression of $d r s$ was significantly induced upon $S$. aureus infection and such state was also observed in the tetracyclinetreated or the extract-treated $S$. aureus-infected groups (Figure 3). Furthermore, the expression of $d r s$ was not statistically significant among all treatments, suggesting that treatments using either tetracycline or roselle calyx extract did not aggravate increased induction of $d r s$. This result indicates that antibacterial activity of tetracycline or roselle calyx extract at the given concentration was not related to the increased production of drosomycin.

Anti-staphylococcal effects of roselle calyx extract in the immunodeficient model system

Improvement on the survival rate of $S$. aureus-infected host and reduction of the in vivo bacterial load upon treatment with roselle calyx extract were most likely not due to the increased production of drosomycin. Therefore, we considered that the protection might be conferred as the result of interaction between antibacterial compounds contained in the extract and $S$. aureus found in infected flies. To further clarify this, we conducted infection experiments on a mutant fly line lacking for Spätzle, an important component in the Toll-mediated humoral immune response against $S$. aureus. As shown in Figure 4, Spätzle-immunodeficient mutant flies succumbed earlier (Figure 4A) with a little higher bacterial load (Figure $4 \mathrm{~B})$ than the genotype control flies (compared to Figure $2 \mathrm{~A}$ and $2 \mathrm{~B}$ ) upon infection with $S$. aureus, clarifying the important role of Toll-mediated protection against $S$. aureus infection. Nevertheless, treatment of S. aureus-

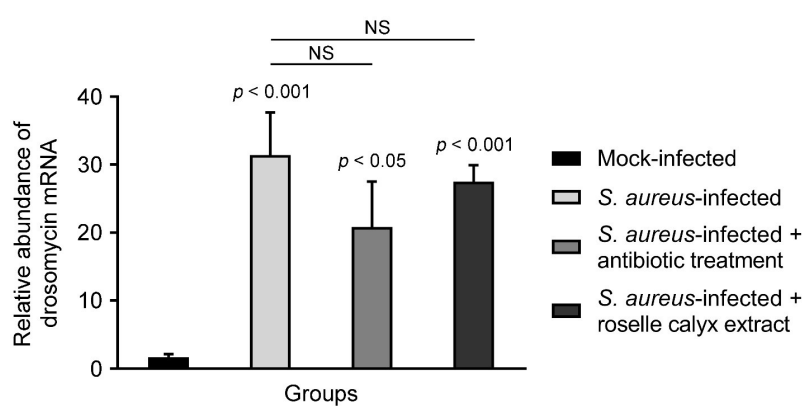

Figure 3. Anti-staphylococcal protection of roselle calyx extract was achieved independent of humoral immunity stimulation. Adult $w^{1118}$ flies at 4-7 days after eclosion were infected with 6 $\times 10^{5} \mathrm{cfu} / \mathrm{ml}$ of $\mathrm{S}$. aureus by pricking, incubated at $25^{\circ} \mathrm{C}$ in the presence of $5 \%$ roselle calyx extract, and subjected to RNA isolation followed by drosomycin mRNA level quantification by RT-qPCR. Expression of reference gene rp49 was used as the internal control.
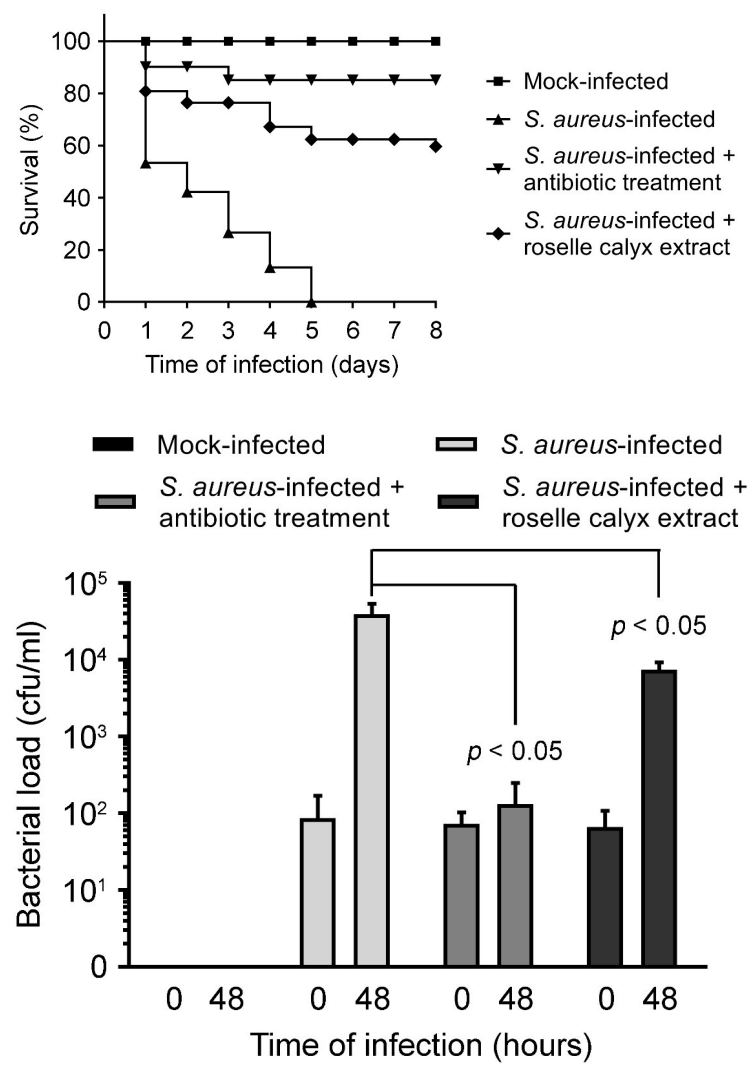

Figure 4. Enhancement of host survivorship and impaired bacterial proliferation in infected immunodeficient $s p z^{\text {mm7/TM6 flies }}$ in the presence roselle calyx extract. Adult $s p z^{\text {rm7/TM6 flies at } 4-7}$ days after eclosion were infected with $6 \times 10^{5} \mathrm{cfu} / \mathrm{ml}$ of $S$. aureus by pricking, incubated at $25^{\circ} \mathrm{C}$ in the presence of $5 \%$ roselle calyx extract, and subjected to fly survival (A) and bacterial load (B) analysis. Flies treated with tetracycline at $200 \mu \mathrm{g} / \mathrm{ml}$ were used as a positive control group.

infected mutant fly line lacking for Spätzle with ethanolic extract of roselle calyx at concentration of $5 \%(\mathrm{w} / \mathrm{w})$ was sufficient to inhibit bacterial proliferation (Figure 4B) and led to the improvement of mutant flies' survivorship 
(Figure 4A). Taken together, our results demonstrated the anti-staphylococcal activity of roselle calyx extract in Drosophila and further clarified that its antibacterial action was not due to the increased activity in Spätzlemediated production of drosomycin.

\section{Discussion}

Infusion prepared from roselle calyces has been generally used by populations from India and countries of Africa as a traditional remedy to treat various illnesses, including infectious diseases (30) and its extract has been shown to yield excellent in vitro antibacterial activities against several bacteria, including $S$. aureus $(12,13)$. However, until now, limited information was available regarding the in vivo anti-staphylococcal activity of this particular preparation. Here, we used a simple and inexpensive in vivo Drosophila platform to assess the anti-staphylococcal activity of roselle calyx extract.

As a well-known member of the gram-positive bacteria, Staphylococcus aureus has been shown to cause extensive negative impacts on the health of many organisms including humans (31) and D. melanogaster (27), thus would be a prospective target for antibacterial drug discovery. Recently, we found that Ulva reticulata extract that has been demonstrated to yield a broad antibacterial activity in the in vitro experiment was able to inhibit $S$. aureus growth in adult Drosophila and this led to a decline in the infected host mortality (14). Taken this into account, we used a similar approach in this research by carrying out straight-forward survival assays and colony forming assays on the $S$. aureus-infected flies in the presence of roselle calyx extract.

Survival assay and colony forming assay are simple yet powerful methods to examine the pharmacological effect of treatment given to live objects that are under certain pressure (in this case, S. aureus infection). From the results, it can be seen that improved survivorship of infected flies was accompanied by the decreased propagation of $S$. aureus in genotype control and immunodeficient mutant fly groups that were treated with food containing roselle calyx extract, suggesting the antibacterial effect of this extract was extended to the individuals in vivo independent on their immune response status and at the same time demonstrating the applicability of survival and colony forming assays to be used together with our established in vivo platform to screen for medicinal plants that are potential as sources to discover antibacterial agents against $S$. aureus.

\section{Conclusion}

In this research, for the first time, we demonstrated the in vivo antibacterial effect of roselle calyx extract against $S$. aureus using a straight-forward and inexpensive Drosophila platform system. In conjunction with currently available in vitro high-throughput screening method, this system would serve as a powerful in vivo approach to clarify the antibacterial activity of not only roselle calyx extract but also numerous medicinal plant extracts prior to be used in further efforts to discover important antibacterial compounds from the corresponding sources. Moreover, this approach will be useful to improve our chance to obtain more appropriate compounds in the initial screening as well as to rule out toxic compounds that are harmful to the eukaryotic cells.

\section{Acknowledgement}

We are grateful to Prof. Yoshinobu Nakanishi of Kanazawa University for the fly lines and Dr. Isra Wahid of Hasanuddin University for his kind help in the determination of total RNA concentration in fly samples. The use of FlyBase is acknowledged.

\section{Authors' contributions}

MA, SS and FN designed the study and conceptualized the experiments, MA, AVG and FN performed the experiments and analyzed the data, MA and FN drafted the manuscript. SS, EW and FN contributed to research materials and supervision of the study. All authors approved the final version of manuscript.

\section{Conflict of Interests}

We declare that we have no conflict of interest.

\section{Ethical Considerations}

Ethical issues (including plagiarism, data fabrication, double publication) have been completely observed by the authors.

\section{Funding/Support}

This research was financially supported by Benua Maritim Indonesia Spesifik (BMIS) research grant (No. 40453/ UN4.1/TU.21/2016) from Hasanuddin University (to F.N.).

\section{References}

1. Gelband H, Petrie MM, Pant S, Gandra S, Levinson J, Barter D, et al. The state of the world's antibiotics. Washington D.C: The Center for Disease Dynamics, Economics \& Policy; 2015.

2. Hadi U, Kuntaman, Qiptiyah M, Paraton H. Problem of antibiotic use and antimicrobial resistance in Indonesia: Are we really making progress? Indones J Trop Infect Dis. 2013;4(4):5-8.

3. Levy SB, Marshall B. Antibacterial resistance worldwide: Causes, challenges and responses. Nat Med. 2004;10:S122-9. doi: 10.1038/ nm 1145 .

4. Luepke KH, Suda KJ, Boucher H, Russo RL, Bonney MW, Hunt TD, et al. Past, present, and future of antibacterial economics: Increasing bacterial resistance, limited antibiotic pipeline, and societal implications. Pharmacotherapy. 2017;37(1):71-84. doi: 10.1002/phar. 1868 .

5. Sommer MOA, Munck C, Kehler RVT, Andersson DI. Prediction of antibiotic resistance: time for a new preclinical paradigm? Nat Rev Microbiol. 2017;15:689-696.

6. WHO. Global antimicrobial resistance surveillance system (GLASS) report. WHO; 2017. Accessed August 23, 2018. 
7. Hassoun A, Linden PK, Friedman B. Incidence, prevalence, and management of MRSA bacteremia across patient populations-a review of recent developments in MRSA management and treatment. Crit Care. 2017;21(1):211. doi: 10.1186/s13054-0171801-3

8. Lewis K. Platforms for antibiotic discovery. Nat Rev Drug Discov. 2013;12:371-387.

9. Abdallah EM. Antibacterial activity of Hibiscus sabdariffa L. calyces against hospital isolates of multidrug resistant Acinetobacter baumannii. J Acute Dis. 2016;5(6):512-516.

10. Khalaphallah R, Soliman WS. Effect of henna and roselle extracts on pathogenic bacteria. Asian Pac J Trop Dis. 2014;4(4):292-6.

11. Gutiérrez-Alcántara EJ, Rangel-Vargas E, Gómez-Aldapa CA, Falfan-Cortes RN, Rodríguez-Marín ML, Godínez-Oviedo A, et al. Antibacterial effect of roselle extracts (Hibiscus sabadariffa), sodium hypochlorite and acetic acid against multidrug-resistant Salmonella strains isolated from tomatoes. Lett Appl Microbiol. 2016;62(2):177-84. doi: 10.1111/lam.12528.

12. Liu KS, Tsao SM, Yin MC. In vitro antibacterial activity of roselle calyx and protocatechuic acid. Phytother Res. 2005;19(11):942-945.

13. Alaga TO, Edema MO, Atayese AO, Bankole MO. Phytochemical and in vitro anti-bacterial properties of Hibiscus sabdariffa $\mathrm{L}$ (Roselle) juice. J Med Plant Res. 2014;8(7):339-344.

14. Nainu F, Asri RM, Arsyad A, Manggau MA, Amir MN. In vivo antibacterial activity of green algae Ulva reticulata against Staphylococcus aureus in Drosophila model of infection. Pharmacog J. 2018;10(5):993-997

15. Buchon N, Silverman N, Cherry S. Immunity in Drosophila melanogaster-from microbial recognition to whole-organism physiology. Nat Rev Immunol. 2014;14:796-810.

16. Hoffmann JA, Reichhart JM. Drosophila innate immunity: An evolutionary perspective. Nat Immunol. 2002;3:121-126.

17. Mussabekova AL, Daeffler L, Imler JL. Innate and intrinsic antiviral immunity in Drosophila. Cell Mol Life Sci. 2017;74(11):2039-2054. doi: 10.1007/s00018-017-2453-9

18. Nainu F. Shiratsuchi A, Nakanishi Y. Induction of apoptosis and subsequent phagocytosis of virus-infected cells as an antiviral mechanism. Front Immunol. 2017;8(1220):1-11. doi: 10.3389/ fimmu.2017.01220.

19. Swevers L, Liu J, Smagghe G. Defense mechanisms against viral infection in Drosophila: RNAi and non-RNAi. Viruses. 2018;10(5):e230.

20. Ugur B, Chen K, Bellen HJ. Drosophila tools and assays for the study of human diseases. Dis Mod Mech. 2016;9(3):235-244.

21. Pandey UB, Nichols CD. Human disease models in Drosophila melanogaster and the role of the fly in therapeutic drug discovery.
Pharmacol Rev. 2011;63(2):411-436.

22. Ekowati H, Arai J, Putri ASD, Nainu F, Shiratsuchi A, Nakanishi Y. Protective effects of Phaseolus vulgaris lectin against viral infection in Drosophila. Drug Discov Ther. 2017;11(6):329-335. doi: 10.5582/ ddt.2017.01071.

23. Fernández-Hernández I, Scheenaard E, Pollarolo G, Gonzalez C. The translational relevance of Drosophila in drug discovery. EMBO Rep. 2016;17(4):471-472.

24. Willoughby LF, Schlosser T, Manning SA, Parisot JP, Street IP, Richardson HE, et al. An in vivo large-scale chemical screening platform using Drosophila for anti-cancer drug discovery. Dis Mod Mech. 2013;6(2):521-9. doi: 10.1242/dmm.009985.

25. Nainu F. Penggunaan Drosophila melanogaster sebagai organisme model dalam penemuan obat. Galenika J Pharm. 2018;4(1):50-67.

26. García-Lara J, Needham AJ, Foster SJ. Invertebrates as animal models for Staphylococcus aureus pathogenesis: A window into host-pathogen interaction. FEMS Immunol Med Microbiol. 2005;43(3):311-323. doi: 10.1016/j.femsim.2004.11.003.

27. Needham AJ, Kibart M, Crossley H, Ingham PW, Foster SJ. Drosophila melanogaster as a model host for Staphylococcus aureus infection. Microbiology. 2004;150(7):2347-55. 10.1099/ mic.0.27116-0

28. Wu K, Conly J, Surette M, Sibley C, Elsayed S, Zhang K. Assessment of virulence diversity of methicillin-resistant Staphylococcus aureus strains with a Drosophila melanogaster infection model. BMC Microbiol. 2012;12(1):274. doi: 10.1186/1471-2180-12-274.

29. Tzelepis I, Kapsetaki S, Panayidou S, Apidianakis Y. Drosophila melanogaster: a first step and a stepping-stone to anti-infectives. Curr Opin Pharmacol. 2013;13(5):763-768. doi: 10.1016/j. coph.2013.08.003.

30. Da-Costa-Rocha I, Bonnlaender B, Sievers H, Pischel I, Heinrich M. Hibiscus sabdariffa L. - A phytochemical and pharmacological review. Food Chem. 2014;165:424-443. doi: 10.1016/j. foodchem.2014.05.002.

31. Tong SYC, Davis JS, Eichenberger E, Holland TL, Fowler VG. Staphylococcus aureus infections: Epidemiology, pathophysiology, clinical manifestations, and management. Clin Microbiol Rev. 2015;28(3):603-661. doi: 10.1128/CMR.00134-14.

32. Ben-Ami R, Watson CC, Lewis RE, Albert ND, Arias CA, Raad II, et al. Drosophila melanogaster as a model to explore the effects of methicillin-resistant Staphylococcus aureus strain type on virulence and response to linezolid treatment. Microb Pathog. 2013;55:1620. doi: 10.1016/j.micpath.2012.11.012.

33. Lemaitre B, Hoffmann J. The host defense of Drosophila melanogaster. Annu Rev Immunol. 2007;25(1):697-743. 